\title{
Magnetoconductance anisotropy of a polymer thin film at the onset of metallicity
}

\author{
S. Zanettini, ${ }^{1}$ J. F. Dayen, ${ }^{1}$ C. Etrillard, ${ }^{1}$ N. Leclerc,${ }^{2}$ M. Venkata Kamalakar, ${ }^{1,3}$ \\ and B. Doudin 1, a) \\ ${ }^{1}$ Institut de Physique et Chimie des Matériaux de Strasbourg (IPCMS), Université de Strasbourg, \\ UMR 7504 CNRS-UdS, 23 rue du Loess, 67034 Strasbourg, France \\ ${ }^{2}$ Institut de Chimie et Procédés pour l'Energie, l'Environnement et la Santé (ICPEES), UMR 7515 CNRS-UdS, \\ 25 rue Becquerel, 67087 Strasbourg, France \\ ${ }^{3}$ Department of Microtechnology and Nanoscience, Chalmers University of Technology, SE-41296 Göteborg, \\ Sweden
}

(Received 11 November 2014; accepted 5 February 2015; published online 12 February 2015)

\begin{abstract}
Thin films of poly(2,5-bis(3-dodecyl-2-yl)-thieno[3,2-b]thiophene) (C12-PBTTT) polymer under electrolyte gating and doping are investigated as model systems for organic thin films devices approaching the metallic side of a metal-insulator (M-I) transition. For the most doped samples, with an estimated density reaching $8 \times 10^{20} \mathrm{~cm}^{-3}$ holes and a conductivity exceeding $1000 \mathrm{~S} \mathrm{~cm}^{-1}$, a positive high-field magnetoconductance is found in a limited temperature range window and only when the field is perpendicular to the sample plane. This signature of weak localization, combined with indications of finite zero-temperature conductivity, allows us to identify delocalized metalliclike transport in these thin films, even though the conductivity decreases when cooling down the samples. C 2015 AIP Publishing LLC. [http://dx.doi.org/10.1063/1.4908526]
\end{abstract}

Metallicity in polymers dates from the discovery of high-conductivity in chemical-doped polymeric materials. ${ }^{1,2}$ Its experimental evidence has however remained elusive, as a hopping conduction mechanism prevails at low temperatures. The lack of a long range crystalline order gives rise to localisation of the electron wave functions and makes the thermally assisted hopping between localized states dominate over the diffusive motion through delocalized states expected in a band-like transport regime. ${ }^{3}$ Very few counterexamples have been reported in chemically highly doped polyaniline $^{1,4,5}$ or polypyrrole bulk materials. ${ }^{2,5}$

Electrolyte gating is a powerful and straightforward approach to apply large transverse electric fields, and possibly dope polymer films. This technique is directly applicable to organic field effect transistor devices, makes possible voltage-tuned filling of the localized tail states, and provides opportunity for metal-like properties to emerge. Claims of metallicity have been reported in electrolyte-gated PBTTTtype thin films through their temperature dependence of the resistivity signature ${ }^{6}$ and electron spin resonance properties, ${ }^{7}$ while a Hall effect indication of delocalized transport has been found in electrolyte-gated P3HT polymer thin film. ${ }^{8}$ However, as recalled by Wang et al.: " the sample disorder (possibly enhanced by the extreme doping conditions) can lead to additional Anderson localization, and impede a clear signature of metallicity, like a finite conductivity when $\mathrm{T} \rightarrow 0$ and the possible occurrence of weak localization." In this letter, we detail our findings on these two experimental signatures.

High-mobility and high-conductivity samples with minimal occurrence of hopping transport are ideally attractive for improving the performance of organic semiconductor thin films, and also highly desired for organic spintronics

a)bdoudin@unistra.fr applications, where the expected long spin lifetimes are motivating scientists to use organic materials for spin manipulation. Solution-processed semiconducting polymers are very attractive from a processing and handling point-ofview, but the significant disorder of thin films favours the insulating side of the M-I transition, and therefore severely limits the distance over which spin coherence persists. ${ }^{9}$ While it would be naive to settle here a long-lasting debate in the solid-state physics community on the definition and signature of metallicity in organic disordered systems, we propose to show in the present work that thin polymer films, relevant for technological electronics applications, can indeed approach the metallic side of the insulator to metal transition, with related expectations of dominant delocalized transport.

Investigations are performed on edge-on oriented C12PBTTT thin films (molecular weight $27 \mathrm{kDa}$, polydispersity of 1.7) obtained by spin coating from ortho-dichlorobenzene solution. ${ }^{10}$ Thin films of $25-30 \mathrm{~nm}$ thickness are spin coated in inert atmosphere on $\mathrm{Si} / \mathrm{SiO}_{2}$ substrates with patterned $30 \mathrm{~nm}$ thick Au electrodes (Fig. 1(a)). The sample geometry allows 4 probes resistance measurements (with two extra side probes for Hall measurements) on a channel length $\mathrm{L}$ of $400 \mu \mathrm{m}$ and a width $\mathrm{W}$ of $100-300 \mu \mathrm{m}$. The picture of the device in Fig. 1(b) also shows the opening window of a $\mathrm{SiO}_{2}$ top coverage $(50 \mathrm{~nm}$ thick) limiting the gate parasitic capacitance and leakage current. Rubber-like ion gel mix of poly(vinylidene fluoride-co-hexafluoropropylene), P(VDF-HFP), and ionic liquid 1-butyl-3-methylimidazolium hexafluorophosphate, [BMIM] $\left[\mathrm{PF}_{6}\right]$, is used as electrolyte gating medium. This choice of ion gel is motivated by the handling convenience and temperature stability. ${ }^{11}$ Polymer processing and ion gel positioning are performed in a glove box enclosure. On/off gating of $10^{4}$ ratio is found between $-2.5 \mathrm{~V}$ and $-3.5 \mathrm{~V}$ bias versus the gate $\mathrm{Au}$ electrode, Fig. 1(c). 
(a)

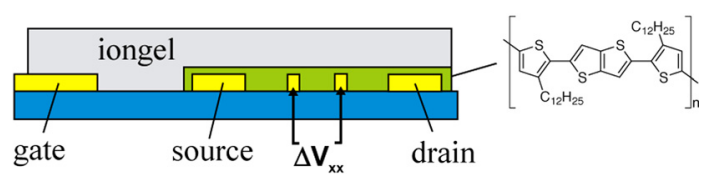

(b)

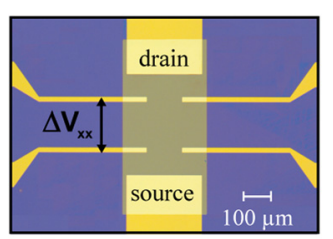

(c)

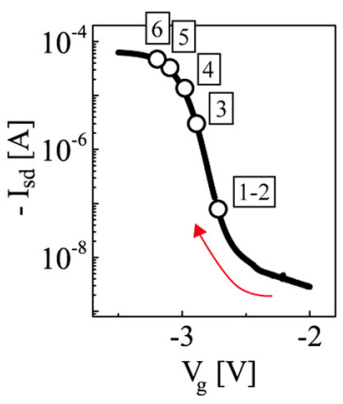

FIG. 1. (a) Side-view of the iongel-gated device, with the chemical structure of C12-PBTTT polymer; (b) optical micrograph of 4-probes (with two supplementary side probes for Hall measurements) device before PBTTT spincoating, where a voltage $\Delta \mathrm{V}_{\mathrm{xx}}$ measures the voltage drop of a current imposed between source and drain electrodes. (c) Transfer curve of an electrolyte-gated C12-PBTTT film, with the arrow indicating the sweeping direction. Dots indicate the gating level of the samples numbered with increasing doping levels.

The significant threshold voltage (for electrolyte-gating standards) is related to a voltage drop due to the limited gate electrode area and its distance from the active channel $(5 \mathrm{~mm})$. The hysteresis of the transfer curve (not shown here), combined with time analysis of the gate current, indicates that polarizing the electrolyte results in significant electrochemical doping of the polymer film, estimated to reach 8 $\times 10^{20} \mathrm{~cm}^{-3}$ holes at $\mathrm{V}_{\mathrm{g}}=-3.5 \mathrm{~V}$. Samples are cooleddown under constant gate voltage; ions in the iongel are immobile below its freezing temperature of $\sim 240 \mathrm{~K}$. We observed that sample cooling stability is critical, with a possible observed resistance discontinuity, typically occurring at $220 \mathrm{~K}$, which makes our room-temperature doping estimates possibly biased at low temperatures. Avoiding cooling under vacuum helps in diminishing this problem, likely related to the adhesion of the ion gel. Such pressure sensitivity has also been reported on ionic liquid gating. ${ }^{12}$

The temperature dependence of the conductivity is the main experimental fingerprint of the dominating charge transport mechanism. Figure 2(a) presents the reduced activation energy $\mathrm{W}$, defined as the logarithmic derivative of the conductivity $W=\mathrm{d}(\ln \sigma) / \mathrm{d}(\ln T)$, for different samples numbered $1 \rightarrow 6$ with increasing doping (see Fig. 1(c)). This logarithmic derivative $\mathrm{W}$ is an unbiased experimental data presentation differentiating between insulating and metallictype behavior. ${ }^{13}$ We observe that the most insulating samples 1 and 2 exhibit the expected increased $\mathrm{W}$ values when cooling down, with a slope of $\ln \mathrm{W}$ versus $\ln \mathrm{T}$ of approximately 0.35 . This slope is reduced for samples 3 and 4 (except a tail stroke of sample 4 at $\mathrm{T}<50 \mathrm{~K}$ ). A temperature independent behavior becomes dominant for the highest doping samples, with $\mathrm{W}$ constant in the whole measured temperature range (1.5-200 K) for samples 5 and 6. A true metallic behavior, with related decreasing $\mathrm{W}$ signature under cooling, is not observed here.

In the insulating side of a M-I transition, the conductivity of the system is governed by thermally activated hopping
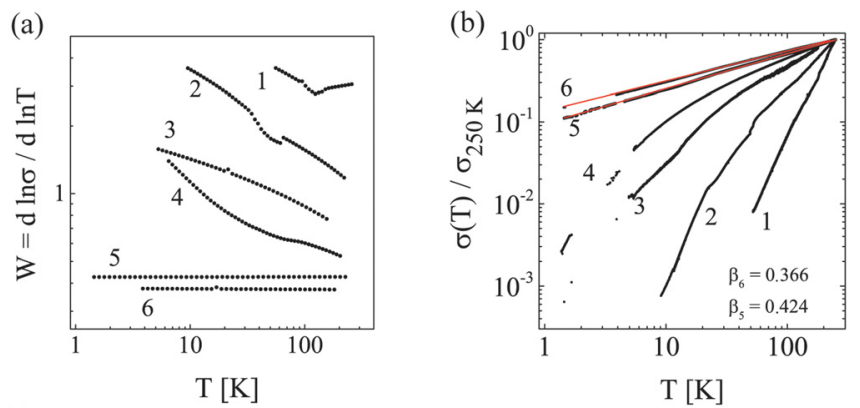

(c)

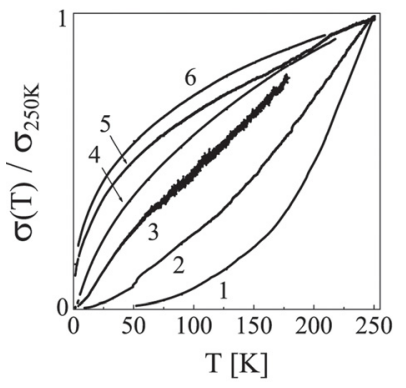

(d)

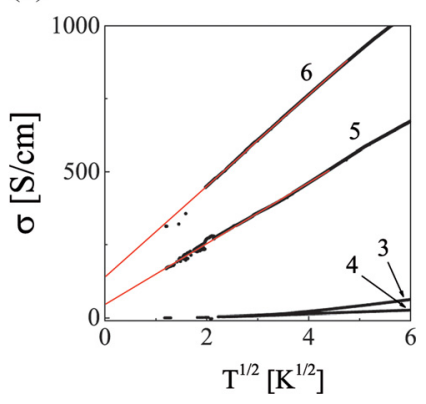

FIG. 2. (a) Reduced activation energy $\mathrm{W}=\mathrm{d}(\ln \sigma) / \mathrm{d}(\ln \mathrm{T})$ for samples $1-6$. (b) Log-Log plot of normalized conductivity (at $250 \mathrm{~K}$ ) vs temperature for samples 1-6; samples 5 and 6 follow a power law function with exponent $\beta$ 0.424 and 0.366 , respectively. (c) Normalized conductivity of samples 1-6 as a function of temperature. Data at highest $\mathrm{T}$ for samples 3,4 , and 6 are affected by conductance discontinuity, thus omitted for clarity. (d) Low temperatures conductivity properties. The horizontal axis scale and zero-temperature extrapolation (in red) follow the power law described in Eq. (3).

between localized states, and the system follows an exponential law, with an exponent that depends on the dimensionality $d$ of the system ${ }^{14}$

$$
\sigma(T)=\sigma_{0} \exp \left[-\left(\frac{T_{M o t t}}{T}\right)^{\frac{1}{d+1}}\right] .
$$

For samples 1 and 2, we find $T_{\text {Mott }}$ temperatures of several thousands Kelvin and an exponent approaching $1 / 3$ that corresponds to $d=2$. This illustrates that our system is quasi two-dimensional (quasi-2D), as expected from the reduced thickness of our polymer thin film $(25-30 \mathrm{~nm})$, which is of the order of estimated coherence length in the $10 \mathrm{~nm}$ range for highly doped polymers. ${ }^{8}$

Figure 2(b) shows that samples 5 and 6 follow a power law dependence of the conductivity, as expected for a conductor in the so-called critical regime, ${ }^{1}$ i.e., approaching the metallic side of a M-I transition

$$
\sigma(T)=\left(e^{2} p_{F} / \hbar^{2}\right)\left(k_{B} T / E_{F}\right)^{1 / \eta} \propto T^{\beta},
$$

where $p_{F}$ is the Fermi momentum, and $1<\eta<3 .{ }^{15}$ The latter is consistent with the fitted values for $\beta$ : $\beta=0.366$ for sample 6 and $\beta=0.424$ for sample 5. It is quite remarkable to observe a simple power law with such a small exponent in the whole range of measured temperatures spanning more than two decades. This strongly suggests that these two samples are at the onset of metallic state, with metallicity fully established when $\beta<0.33$. ${ }^{1,16}$

The normalized plot in Figure 2(c) emphasizes the transition from convex conductivity versus temperature curves for insulating-type samples, to concave curves found for 
highly conductive samples. This relates to the change of the second derivative $\sigma^{\prime \prime}(\mathrm{T})$ sign when moving from the insulator towards the transition to the metallic side, as deduced from Eqs. (1) and (2) (if $\beta<1$ ). Interestingly, we found that this presentation of the data is illustrating at best the contrast between the two types of samples. Note that the noise in the data significantly exceeds the measurement setup noise at the intermediate case 3 (Fig. 2(c)).

The samples behavior when $\mathrm{T} \rightarrow 0$ is studied in more details in Fig. 2(d), revealing that the highest conductivity samples 5 and 6 are characterized by a positive value of the extrapolated $T=0 \mathrm{~K}$ value on the $\mathrm{y}$-axis, indicative of a finite zero temperature conductivity. The conductivity temperature dependence for a disordered metal is described by the relationship ${ }^{3}$

$$
\sigma(T)=\sigma_{0}+a T^{1 / 2}+b T^{p / 2},
$$

where the second term is the lowest order correction to the conductivity arising from electron-electron interaction and the third term is determined by the correction to $\sigma_{0}$ due to the weak-localization effects. At low temperatures, phonon scattering is strongly reduced and the main dephasing mechanism is electron-electron interactions. Moreover, theoretical calculations show that the exponent $p$ reaches 1 when approaching the metallic side of the transition, ${ }^{17}$ justifying the $\sigma-\mathrm{T}^{1 / 2}$ approximation in Fig. 2(d). The intercept with the $T=0 \mathrm{~K}$ axis becomes larger (respectively, $\sigma_{0} \sim 40 \mathrm{~S} \mathrm{~cm}^{-1}$ for sample 5 and $150 \mathrm{~S} \mathrm{~cm}^{-1}$ for sample 6) for increasingly conductive samples, while the zero temperature conductivity is negligible for samples on the insulating side of the transition. The finite zero-temperature conductivity is considered to be the key experimental signature of metallicity. Such indication in Fig. 2(d) is quite remarkable for samples having a non-decreasing resistivity under cooling. Note that finite $0 \mathrm{~K}$ conductivity and a temperature power law of the conductivity are also predicted for a nuclear-mediated polaron hopping model. ${ }^{18} \mathrm{We}$ find however that our exponent values are smaller by a factor of 3-20 than those reported in Ref. 18. Our high conductivity would also result in unusually long hopping lengths under the hypothesis of Ref. 18. In the following, we show how magnetoconductance (MC) studies confirm the expectation (common to both models) that significantly large phase coherence length can occur in our samples.

For conducting polymer thin films approaching the metallic side of the M-I transition, both electron-electron interactions and weak localization effects are expected to coexist in the MC response, providing contributions of opposite signs: a magnetic field suppresses the quantum interference processes related to weak localization of charge carriers, leading therefore to positive MC; electron-electron interactions lead to negative MC, and are expected to become dominant when decreasing the temperature. ${ }^{3,19}$ If the carriers trajectories are mostly planar, as expected in our anisotropic thin film, disruption of weak localization should only occur when the applied field is normal to the plane, in contrast to the isotropic electron-electron interactions contribution. Temperature studies of the anisotropy of MC properties of the highly conductive samples are therefore performed (Fig. 3).

The occurrence of positive MC of a few percent (Fig. 3(a)) suggests that the most conductive samples have accessed a metallic-like type of transport in which the coherence length is long enough to give rise to quantum interference effects. This effect disappears at high temperatures, as expected when phase-breaking scattering events become dominant. In the lowest temperature range, the positive MC disappears and a significant negative MC dominates (Fig. $3(\mathrm{c})$ ). This crossover is expected as electron-electron interactions start taking place at the lowest temperatures. For sample 5 the crossover temperature is around $4.5 \mathrm{~K}$, with a slightly lower transition temperature for sample 6, still exhibiting positive $\mathrm{MC}$ signature at $3 \mathrm{~K}$ (not shown here). Note that the noise of the MR properties in Fig. 3 also (a)

H perpendicular

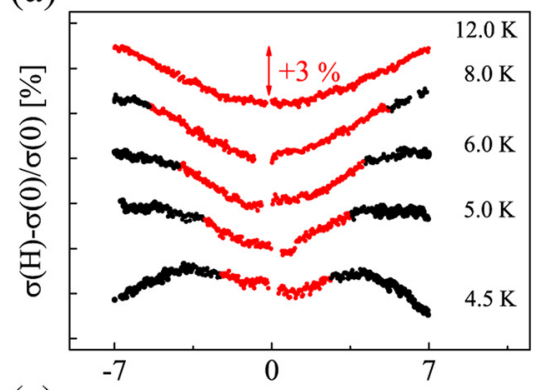

(c)

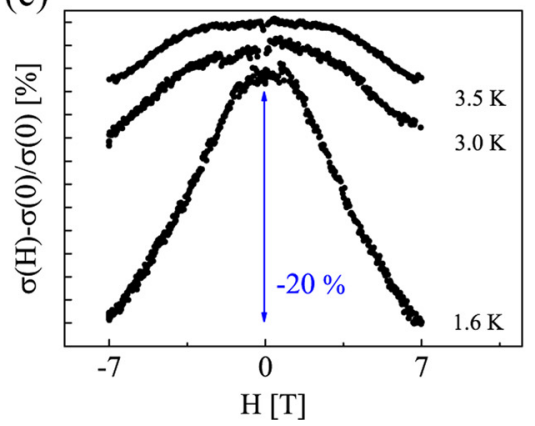

(b)

H parallel

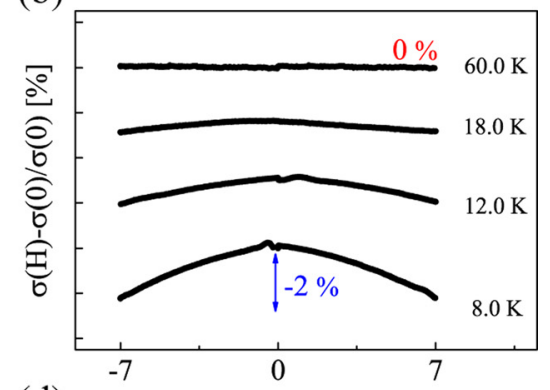

(d)

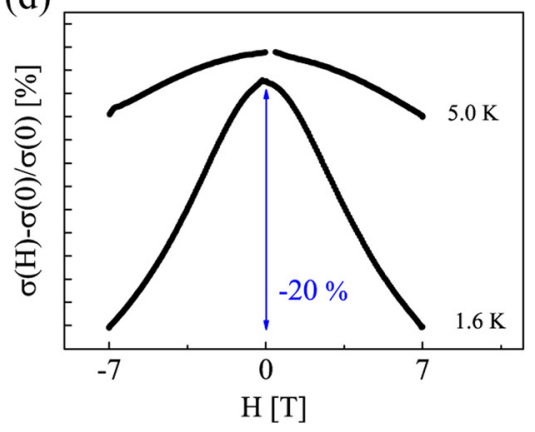

FIG. 3. Magnetoconductivity (\%) of a highly doped C12-PBTTT thin film approaching the metallic side of the M-I transition, studied as a function of temperature. Field normal to the film plane for (a) and (c) and parallel to the film plane for (b) and (d) (parallel to source-drain current), with shifted zero of the curves for added clarity. We separated the curves in two temperature ranges to show clearly a change of regime, from isotropic at the lowest temperatures (b) and (d), to anisotropic when heating the samples (a) and (c). 
appears anisotropic. Such peculiarity, possibly related to the quoted intrinsic sample noise mentioned in Fig. 2, deserves more systematic studies, beyond the scope of this letter. Positive MC has been reported for thick bulk-like chemically doped poly(acetylene),${ }^{20}$ poly( $p$-phenylenevinylene),${ }^{21}$ and PANI-AMPSA, ${ }^{22}$ all suggesting the occurrence of weak localization. Such a clear temperature transition between the two regimes has not been reported for organic systems. Our thin quasi-2D samples also provide a unique opportunity to study anisotropy properties of MC.

Figs. 3(b) and 3(d) represent MC in the case of magnetic field parallel to the sample plane, more specifically parallel to the source-drain current direction. At low temperatures, the $\mathrm{MC}$ is essentially isotropic, as expected from isotropic e-e interactions. The complete absence of positive MC up to $60 \mathrm{~K}$ (Fig. 3(b)) illustrates the anisotropy of MC properties. This is in agreement with the strongly anisotropic nature of the sample, for which a normal magnetic field orientation is much more efficient for breaking the constructive interference of self-closing paths. We therefore consider that the anisotropy of the positive MC, as well as its limited temperature range occurrence (limited by electron-electron interactions at lowest temperatures and phonon-electron coupling at higher temperatures) strongly point towards the occurrence of weak localization effects.

For disordered electronic systems, the electron-electron interaction decreases the conductivity when applying the magnetic field due to the Zeeman splitting of bands, with a related change of conductivity ${ }^{3,18}$

$$
\begin{aligned}
& \Delta \sigma(H, T)=-0.041\left(g \mu_{B} / k_{B}\right) \alpha_{d} \gamma F_{\sigma} T^{-3 / 2} H^{2}, \\
& \quad \text { for } \mathrm{g} \mu_{\mathrm{B}} \mathrm{H} \ll \mathrm{k}_{\mathrm{B}} \mathrm{T}, \\
& \Delta \sigma(H, T)=\alpha_{d} \gamma F_{\sigma} T^{1 / 2}-0.77 \alpha_{d}\left(g \mu_{B} / k_{B}\right)^{1 / 2} \gamma F_{\sigma} H^{1 / 2}, \\
& \quad \text { for } \mathrm{g} \mu_{\mathrm{B}} \mathrm{H} \gg \mathrm{k}_{\mathrm{B}} \mathrm{T} .
\end{aligned}
$$

We illustrate in Fig. 4 that the low (respectively, high) field conductivity indeed follows a $H^{2}$ (respectively, $H^{1 / 2}$ ) power law, confirming the hypothesis of dominant electron-electron interactions below $4.5 \mathrm{~K}$.

In conclusion, highly conductive C12-PBTTT results from electrolyte gating, with values between $1200 \pm 200 \mathrm{~S} /$

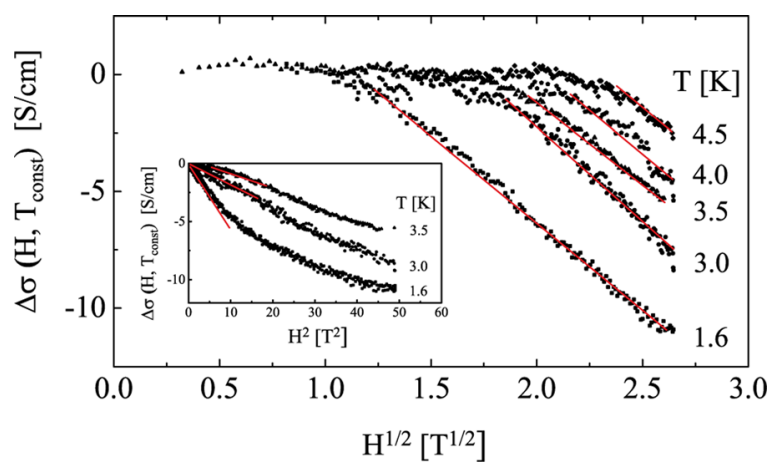

FIG. 4. Magnetoconductivity as a function of $\mathrm{H}^{1 / 2}$ at several temperatures, with linear dependence expected at high field (underlined by the red lines) for e-e interactions model. Inset: Conductivity as a function of $\mathrm{H}^{2}$ at several temperatures with linear dependence expected at low field. The magnetic field is normal to the sample plane. Data are the same as Figs. 3(a) and 3(c). $\mathrm{cm}$ and $1600 \pm 200 \mathrm{~S} / \mathrm{cm}$ for the most doped samples (respectively, 5 and 6). They exhibit the finite lowtemperature conductivity and weak localization experimental fingerprints of a quasi-2D disordered metallic thin film. While our findings do not exclude a nuclear-mediated hopping mechanism with very long (several tens of $\mathrm{nm}$ ) hopping distance, they call for more calculations and studies of magnetoresistance properties in such model. One should emphasize that our findings are of particular importance when considering the type of materials, with processing and thin film geometry directly relevant for applications, such as transistor devices. A strategy of electrolyte doping of thin polymer films can be of high interest for organic spin valve devices as well. Increasing scattering or hopping lengths, and providing a better resistance match between active channel and electrodes could make significant electrical spin injection and detection with metallic magnetic electrodes possible.

We thank C. Leighton and T. Heiser for fruitful discussions, and F. Chevrier for technical help. Partial financial support of the Agence Nationale de la Recherche (MULTISELF 11-BS08-06, Labex NIE 11-LABX0058_NIE within the Investissement d'Avenir program ANR-10-IDEX-0002-02), the International Center for Frontier Research in Chemistry (icFRC, Strasbourg), and the technical support of the STnano cleanroom are also gratefully acknowledged.

${ }^{1}$ R. Menon, C. Yoon, D. Moses, and A. Heeger, Phys. Rev. B 48, 17685 (1993).

${ }^{2}$ C. Yoon, D. Moses, and A. Heeger, Phys Rev. B 49, 10851 (1994).

${ }^{3}$ P. A. Lee and T. V. Ramakrishnan, Rev. Mod. Phys. 57, 287 (1985).

${ }^{4}$ K. Lee, S. Cho, S. H. Park, A. J. Heeger, C. W. Lee, and S. H. Lee, Nature 441, 65 (2006).

${ }^{5}$ R. S. Kohlman, A. Zibold, D. B. Tanner, G. G. Ihas, T. Ishiguro, Y. G. Min, A. G. MacDiarmid, and A. J. Epstein, Phys. Rev. Lett. 78, 3915 (1997).

${ }^{6}$ A. S. Dhoot, J. D. Yuen, M. Heeney, I. McCulloch, D. Moses, and A. J. Heeger, Proc. Nat. Acad. Sci. U.S.A. 103, 11834 (2006).

${ }^{7}$ H. Tanaka, M. Hirate, S. Watanabe, and S. I. Kuroda, Adv. Mater. 26, 2376 (2014).

${ }^{8}$ S. Wang, M. Ha, M. Manno, C. D. Frisbie, and C. Leighton, Nat. Commun. 3, 1210 (2012).

${ }^{9}$ G. Szulczewski, S. Sanvito, and M. Coey, Nature Mater. 8, 693 (2009).

${ }^{10}$ L. Biniek, N. Leclerc, T. Heiser, R. Bechara, and M. Brinkmann, Macromolecules 46, 4014 (2013).

${ }^{11}$ K. H. Lee, M. S. Kang, S. Zhang, Y. Gu, T. P. Lodge, and C. D. Frisbie, Adv. Mater. 24, 4457 (2012).

${ }^{12}$ W. Shi, J. Ye, J. G. Checkelsky, C. Terakura, and Y. Iwasa, Adv. Funct. Mater. 24, 2005 (2014).

${ }^{13}$ A. G. Zabrodskii and K. N. Zinov'eva, Zh. Eksp. Teor. Fiz. 86, 727 (1984) [Sov. Phys. JETP 59, 345 (1984)].

${ }^{14}$ B. I. Shklovskii and A. L. Efros, Electronic Properties of Doped Semiconductors (Springer-Verlag, 1984).

${ }^{15}$ W. L. McMillan, Phys. Rev. B 24, 2739 (1981).

${ }^{16}$ M. Ahlskog, R. Menon, and A. J. Heeger, J. Phys. Condens. Matter 9, 4145 (1997).

${ }^{17}$ D. Belitz and K. I. Wysokinski, Phys. Rev. B 36, 9333 (1987).

${ }^{18}$ K. Asadi, A. J. Kronemeijer, T. Cramer, L. J. A. Koster, P. W. M. Blom, and D. M. de Leeuw, Nat. Commun. 4, 1710 (2013).

${ }^{19}$ A. N. Aleshin, Phys. Solid State 52, 2307 (2010).

${ }^{20}$ K. Väkiparta, R. Menon, M. R. Andersson, Y. Cao, D. Moses, and A. J. Heeger, Synth. Met. 57, 4860 (1993).

${ }^{21}$ M. Ahlskog, R. Menon, A. J. Heeger, T. Noguchi, and T. Ohnishi, Phys. Rev. B 53, 15529 (1996).

${ }^{22}$ A. K. Mukherjee and R. Menon, J. Phys. Condens. Matter 17, 1947 (2005). 